\title{
Spatial acuity and summation on the hand: The role of thermal cues in material discrimination
}

\author{
Gi-Hun YANG \\ Korea Institute of Science and Technnology, Seoul, Korea \\ Dong-Soo KwoN \\ Korea Advanced Institute of Science and Technology, Daejeon, Korea \\ AND \\ LyNETTE A. JONES \\ Massachusetts Institute of Technology, Cambridge, Massachusetts
}

\begin{abstract}
The spatial characteristics of thermal perception were studied in two experiments that examined how thermal stimuli are processed within the hands. A thermal display that simulates cues associated with making contact with different materials was used in these studies. In the first experiment, participants indicated which of two simulated materials that were presented to the index fingertip was cooler. The results indicated that participants were unable to resolve the two areas of thermal stimulation. In the second experiment, the effects of concurrent thermal stimulation on the ability to discriminate between simulated materials were evaluated. Thermal cues were presented to the middle fingers of both hands and to two adjacent fingers on one hand. Thermal spatial summation was evident across the fingers, which enhanced the ability to discriminate between materials when the cooler stimulus was presented to three fingers. When the same stimulus was presented to the two hands, the stimulation of adjacent fingers altered the perceived thermal response.
\end{abstract}

When an object is grasped in the hand, thermal cues provide information about the object's temperature and thermal properties, which can be used to assist in identifying it. Because the fingers are usually warmer than objects encountered in the environment, the perception of temperature is based on the responses of cold thermoreceptors in the skin that respond to decreases in skin temperature. The rate at which the skin changes temperature on contact depends on both the initial temperatures of the hand and object, and their thermal properties, such as conductivity and heat capacity. Several studies have shown that people can identify materials on the basis of thermal cues alone (Caldwell \& Gosney, 1993; Ho \& Jones, 2007; Ino et al., 1993), although their ability to do so depends on the thermal properties of the materials being identified. Materials with lower thermal effusivities (see Table 1), such as foam or plastic, are easier to identify than those with higher effusivities, such as stainless steel or copper (Ho \& Jones, 2007).

A number of thermal displays have been built and tested to evaluate the role of thermal cues in object identification and discrimination (see, e.g., Caldwell, Tsagarakis, \& Wardle, 1997; Ino et al., 1993; Jones \& Berris, 2003). These systems typically comprise a thermal heater/cooler (Peltier device), temperature sensors, and a temperature control system that monitors and controls the surface temperature of the display (for a review, see Jones \& Ho, 2008). For some of these displays, experimental data on the changes in skin temperature when the hand makes contact with a real material have been used to simulate thermal changes (Caldwell et al., 1997; Ino et al., 1993), whereas for others a thermal model has been used to characterize the heat transfer process between the skin and an object during contact (see, e.g., Benali-Khoudja, Hafez, Alexandre, \& Kheddar, 2003; Ho \& Jones, 2006a). The thermal responses required to simulate materials are then calculated from the model on the basis of the material's properties and initial conditions. In general, these thermal displays can provide thermal cues that allow participants to either identify materials or discriminate between them with a success rate that parallels their performance with real materials (Caldwell \& Gosney, 1993; Ho \& Jones, 2007; Ino et al., 1993).

Multifingered thermal displays have also been developed to study the spatial characteristics of thermal perception within and across the hands so that effective thermal cues can be created in virtual environments (Caldwell \& Gosney, 1993; Deml, Mihalyi, \& Hannig, 2006; Yang, Jones, \& Kwon, 2008). The focus of this research has been on determining the contribution of thermal cues to haptic

D.-S. Kwon, kwonds@kaist.ac.kr 
Table 1

Thermophysical Properties of the Simulated Materials (Matweb, 2007)

\begin{tabular}{lrcrr}
\hline \multicolumn{1}{c}{ Materials } & $\begin{array}{r}\text { Density } \\
\left(\mathrm{kg} / \mathrm{m}^{3}\right)\end{array}$ & $\begin{array}{c}\text { Thermal } \\
\text { Conductivity } \\
(\mathrm{W} / \mathrm{m} \cdot \mathrm{K})\end{array}$ & $\begin{array}{c}\text { Specific } \\
\text { Heat } \\
(\mathrm{J} / \mathrm{kg} \cdot \mathrm{K})\end{array}$ & $\begin{array}{c}\text { Thermal } \\
\text { Effusivity } \\
(\mathrm{k \rho c})^{1 / 2}\end{array}$ \\
\hline Urethane & 70 & 0.026 & 1,045 & 43.6 \\
Wood & 545 & 0.17 & 2,385 & 470 \\
Glass & 2,500 & 1.4 & 750 & 1,620 \\
Stainless steel & 8,000 & 13.5 & 460 & 7,048 \\
Copper & 8,933 & 401 & 385 & 37,136 \\
\hline
\end{tabular}

object recognition and on how discrete thermal stimuli are processed within the hand. For example, Yang et al. (2008) found that the ability to identify materials on the basis of thermal cues improved when thermal stimuli were presented to three as compared with one finger, but that there was little further improvement in performance when five fingers were stimulated. These studies have highlighted the importance of understanding how thermal sensory information from different parts of the hand is processed within the central nervous system.

In this article, two experiments on thermal perception are described that focus on how the spatial features of the thermal stimulus influence perception. In the first experiment, the thermal spatial acuity of the fingertip was evaluated by simulating the changes in skin temperature that would occur as the finger makes contact with different materials. The second experiment examined the effect of thermal stimulation of the adjacent fingers on the perception of temperature changes on a target finger. In these experiments, we sought to elucidate the characteristics of thermal spatial acuity and summation on the fingers using thermal stimuli that are typical of those encountered during haptic exploration of objects.

\section{Thermal Model}

To simulate the contact between the fingers and an object in a thermal display, a model is required that characterizes the heat transfer between the object and fingers. A number of models have been proposed, most of which are based on a transient heat conduction process (Benali-Khoudja et al., 2003; Bergamasco, Alessi, \& Calcara, 1997; Ho \& Jones, 2007; Kammermeier, Kron, Hoogen, \& Schmidt, 2004; Yamamoto, Cros, Hashimoto, \& Higuchi, 2004; Yang, Kyung, Jeong, \& Kwon, 2005). Several of these authors (Ho \& Jones, 2006a; Yamamoto et al., 2004) used a simple, semi-infinite body model to simulate the contact between the finger and an object, and the model appeared to simulate the various materials well. Two variables that were not considered in these models were thermal contact resistance and blood perfusion, both of which are included in the present model. The proposed model is based on the semi-infinite body model (Incropera \& DeWitt, 1996), and it is changed slightly using Equation 1 and Boundary Conditions 2 and 3 (see below), where $k_{f}$ and $k_{\mathrm{obj}}$ indicate the thermal conductivity of the skin and object, respectively; $\rho_{f}$ and $\rho_{\mathrm{obj}}$ denote the density of the skin and object, respectively; $c_{f}$ and $c_{\text {obj }}$ signify the heat capacity of the skin and object, respectively; $R_{t . c}$ indicates the surface thermal contact resistance; and $e_{f}$ and $e_{\mathrm{obj}}$ indicate the thermal effusivity of the finger and object, respectively (Yamamoto et al., 2004). The latter term is also called the contact coefficient $\left[(k \rho c)^{1 / 2}\right]$ (Businger \& Buettner, 1961). Thermal diffusivity is calculated as

$$
\alpha=\frac{k}{\rho c} \text {. }
$$

Using the Laplace transform method, the temperature profile of the fingertip is obtained from Equation 4 (see below).

It was assumed that the temperature of the fingertip in a normal, healthy person is approximately $32^{\circ} \mathrm{C}$, and that the ambient temperature-and hence the object's temperature-is $24^{\circ} \mathrm{C}$. When an object makes contact with a fingertip, heat is conducted out of the skin, and the decrease in skin temperature that is associated with this process is used to assist in identifying the object. When

$$
\begin{gathered}
k_{f} \frac{\partial^{2} T}{\partial^{2} x}=\rho_{f} c_{f} \frac{\partial T_{f}}{\partial t} \quad k_{\mathrm{obj}} \frac{\partial^{2} T_{\mathrm{obj}}}{\partial x^{2}}=\rho_{\mathrm{obj}} c_{\mathrm{obj}} \frac{\partial T_{\mathrm{obj}}}{\partial t} \\
-\left.k_{f} A \frac{\partial T_{f}}{\partial x}\right|_{x=0}+\bar{h}\left(T_{\mathrm{blood}}-T_{f}(0, t)\right)=\left.k_{\mathrm{obj}} A \frac{\partial T_{\mathrm{obj}}}{\partial x}\right|_{x=0}=\frac{T_{f}(0, t)-T_{\mathrm{obj}}(0, t)}{R_{t . c}} \\
-\left.k_{f} \frac{\partial T_{f}}{\partial x}\right|_{x=\infty}=0 \quad-\left.k_{\mathrm{obj}} \frac{\partial T_{\mathrm{obj}}}{\partial x}\right|_{x=\infty}=0 \\
T_{f}(x, t)=J\left\{\frac{1}{K} \operatorname{erfc}\left(\frac{x}{2 \sqrt{\alpha_{f} t}}\right)-\frac{1}{K} e^{\left(K x+\alpha_{f} K^{2} t\right)} \operatorname{erfc}\left(\frac{x}{2 \sqrt{\alpha_{f} t}}+K \sqrt{\alpha_{f} t}\right)\right\}+T_{\text {skin }} \\
e_{\mathrm{obj}}=\sqrt{k_{\mathrm{obj}} \rho_{\mathrm{obj}} c_{\mathrm{obj}}} \quad e_{f}=\sqrt{k_{f} \rho_{f} c_{f}} \quad K=-\frac{1}{k_{f}}\left\{\bar{h}+\frac{1}{R_{t . c}}\left(\frac{e_{f}+e_{\mathrm{obj}}}{e_{\mathrm{obj}}}\right)\right\}
\end{gathered}
$$




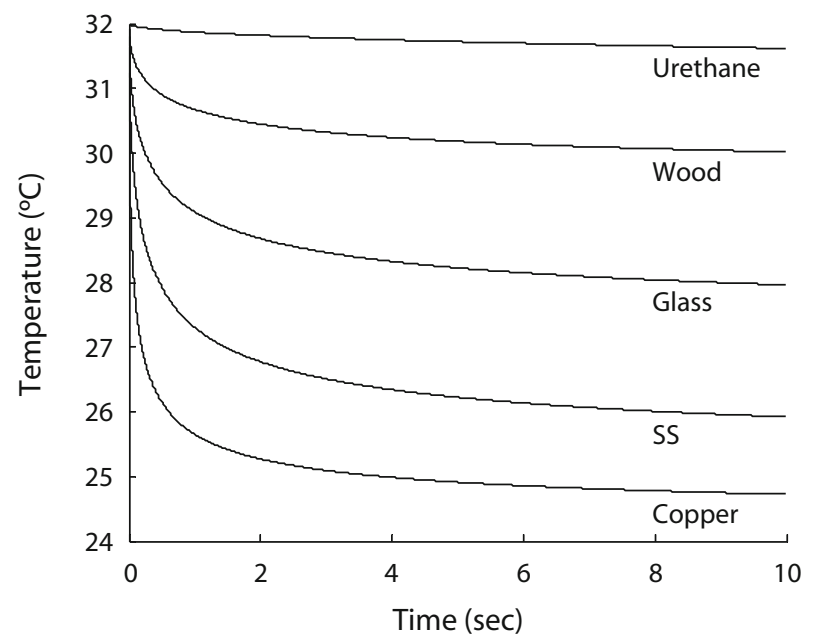

Figure 1. Model predictions for the decrease in skin temperature when the fingertip makes contact with materials with varying thermophysical properties. SS, stainless steel.

only the heat conduction between the object and fingertip is considered, heat transfer can be modeled using either a lumped capacitance model or a heat diffusion equation. If the contact time is brief and the Fourier number of the material is small, a semi-infinite body model describes the transient conduction process between the object and fingertip well (Ho \& Jones, 2006a). However, in a further study, Ho and Jones (2007) noted that the decreases in skin temperature were smaller than those predicted by the semi-infinite body model. They proposed that blood perfusion and thermal contact resistance may have to be considered in the heat-transfer process between an object and the fingertip. These two factors were therefore included in the heat-transfer model used in the present experiments. Thermal contact resistance has been calculated by Yovanovich (1986) and Ho and Jones (2006b), and the parameters are also referred to in Benali-Khoudja et al. (2003). On the basis of those data, the response of the fingertip to contact with five different materials (urethane, wood [oak], glass, stainless steel [SS], and copper) was simulated. The simulations are illustrated in Figure 1.

\section{Thermal Display}

A thermal feedback display that can stimulate up to five fingers simultaneously was used in these experiments. The display consists of thin-film resistance temperature detectors (RTDs, HansTech Co.), water-cooling jackets, and Peltier thermoelectric modules, as shown in Figure 2. The response times (RTs) of the RTDs are extremely short-on the order of several milliseconds-depending on the magnitude of the temperature change. The temperatures of the Peltier devices in the thermal display were controlled in real time with a digital PI control loop that was implemented at $1 \mathrm{kHz}$ on a data acquisition board (Sensoray626). Current amplifiers (boosters) were used to control each Peltier module independently. Effective cooling was achieved by attaching a small water-cooling jacket to the heated side of each Peltier device. The cooling jackets were connected to a recirculating chiller (Model F12-ED, JULABO). During thermal stimulation, a Visual $\mathrm{C}++$ program recorded the participants' responses, and the temperatures of the surfaces of the Peltier devices were sampled at $100 \mathrm{~Hz}$. Each Peltier module is $15 \times$ $15 \times 4 \mathrm{~mm}$, with a maximum heat load $\left(\mathrm{Q}_{\max }\right)$ of $6 \mathrm{~W}$ and a maximum electrical input power of $9 \mathrm{~W}$. These devices have a cooling rate of $6^{\circ} \mathrm{C} / \mathrm{sec}$ and a heating rate of $12^{\circ} \mathrm{C} /$ $\mathrm{sec}$ and can display temperatures ranging from $0^{\circ}$ to $60^{\circ} \mathrm{C}$. Because the positions of the fingertips vary with hand size, the thermal display was designed so that the position of each thermal stimulator could be adjusted.

\section{EXPERIMENT 1 Thermal Spatial Acuity}

\section{Method}

Participants. Six normal, healthy individuals $(5$ men and 1 woman) 23-30 years of age participated in this experiment. They had no known abnormalities of their tactile or thermal sensory systems and had no history of peripheral vascular disease. This research was approved by the KAIST Ethics Committee.

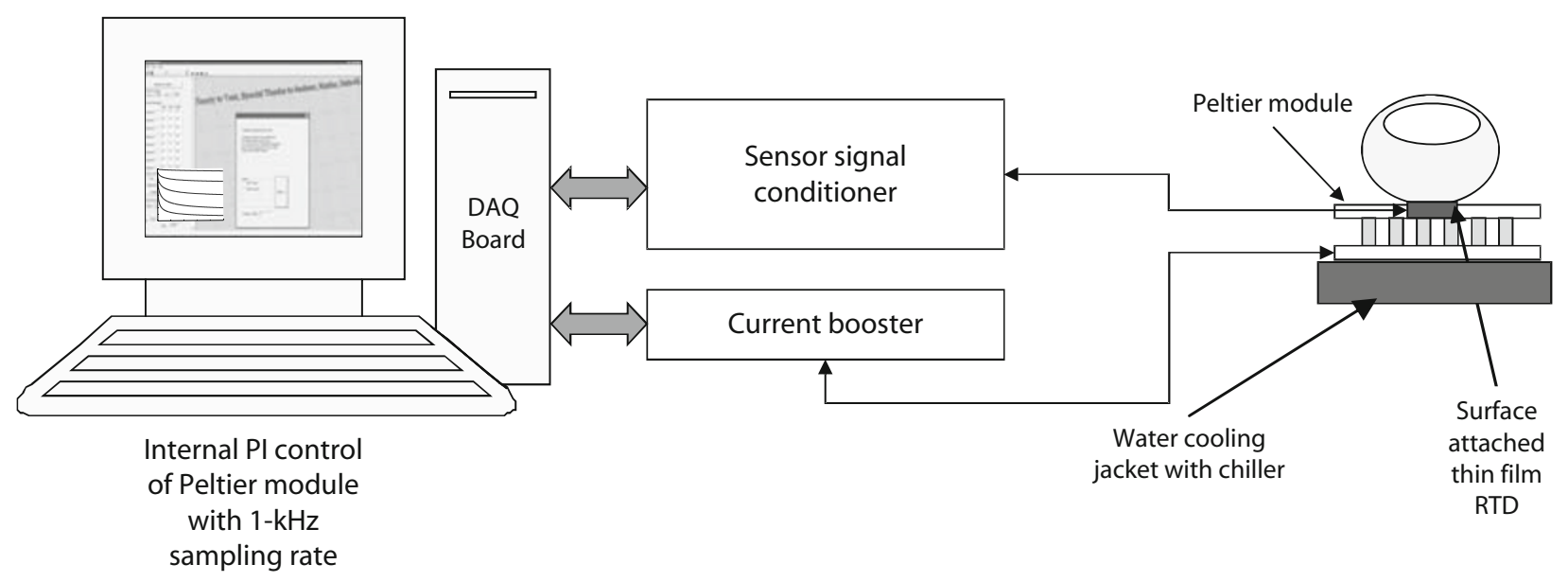

Figure 2. Schematic diagram of the thermal feedback device. 


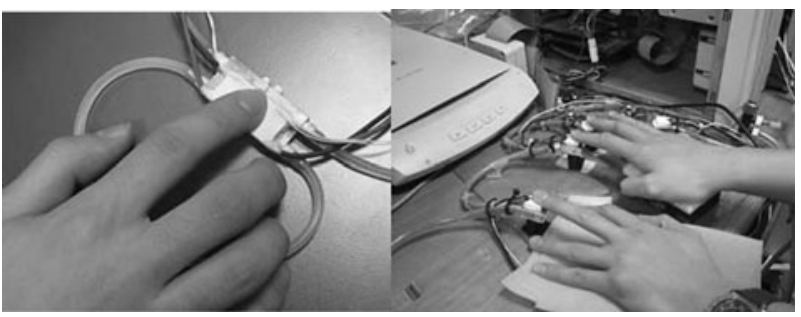

Figure 3. Thermal feedback display modules used in the spatial acuity (left) and spatial summation (right) experiments.

Apparatus. The following five materials, which cover a broad range of thermal properties, were simulated in this experiment: urethane, wood (oak), glass, SS, and copper. Their properties are listed in Table 1. The simulated thermal cues calculated from the semiinfinite body model were displayed on the surfaces of two Peltier modules. The Peltier devices were positioned immediately adjacent to each other in order to create a continuous surface measuring $30 \times$ $15 \times 4 \mathrm{~mm}$, as shown in Figure 3. The gap between the two devices was $0.15 \mathrm{~mm}$. Each Peltier module presented a simulated material and was controlled independently, which ensured that the responses of each Peltier device were accurate.

Procedure. Prior to the experiment, the contact area was determined from images that were taken of the finger after it was moistened and made contact with the surfaces of the Peltier devices coated with baking soda. The contact area of the fingertip on the display was $110 \mathrm{~mm}^{2}$; thus, each thermal stimulus was approximately $55 \mathrm{~mm}^{2}$. At the start of each trial, participants placed their right index fingertip on the surface of the thermal display centered over the two Peltier modules (see Figure 3). The surface temperatures of the Peltier devices were initially maintained at $32^{\circ} \mathrm{C}$. In each trial, one of eight possible pairs of simulated materials was randomly displayed. The eight stimulus combinations (SS-urethane, SS-wood, SS-glass, copperurethane, copper-wood, copper-glass, copper-SS, glass-wood) were selected so that the differences between their thermal properties were maximized. These eight combinations were each presented four times in a random order, giving a total of 32 trials. A three-alternative forced choice (3AFC) method was adopted in which participants were instructed to indicate after $10 \mathrm{sec}$ whether the left or right side of the display was cooler or whether the surface was the same (i.e., a uniform temperature). After $10 \mathrm{sec}$, participants removed their finger from the stimulation surfaces and indicated their responses. There was at least $15 \mathrm{sec}$ between trials, and after every block of 7 trials, there was a 3 -min rest period. This ensured that both the skin temperature and the temperature of the display returned to control levels between trials. Participants could also request a break whenever necessary during the experiment. The participants' answers were recorded using an experimental program written in Visual $\mathrm{C}++$. The surface temperatures of the Peltier devices were recorded in every trial.

\section{Results}

In Experiment 1, the responses were analyzed in terms of the number of correct responses - that is, successful identification of the "cooler" of the two simulated materials, as defined in terms of the predicted thermal responses based on the semi-infinite body model. The chance level was $33 \%$, and a threshold level of $72 \%$ correct responses was chosen as indicating that participants could reliably discriminate between a pair of materials. The percentage of correct responses for the various combinations of materials is shown in Table 2. As can be seen in this table, participants were not able to discriminate reliably between the various simulated materials, and for the combinations presented, all thresholds were below $72 \%$, with a mean of $28.7 \%$ correct. The overall percentage of "same" responses was $55.7 \%$. The five materials were chosen to span a range of thermal effusivities $\left[(k \rho c)^{1 / 2}\right]$, and for these simulated materials, the smallest ratio of thermal effusivities was 3.4:1 (wood and glass).

When the results from this experiment are compared with those from an earlier study in which the same two simulated materials were presented to the left and right index fingers (Yang et al., 2008), the decline in performance is immediately apparent. As can be seen in Figure 4, when the simulated thermal stimuli are presented to the two hands, participants are able to discriminate between them with an average response rate of $90 \%$ correct. When the same stimuli are presented to a smaller surface area within a finger, participants are unable to discriminate between them.

\section{Discussion}

The results from this experiment indicate that participants were not able to discriminate between two thermal stimuli presented to the fingertip of a single finger. The particular materials simulated were chosen to span a broad range of thermophysical properties, as can be seen in Table 1. In this study, it was found that even when the ratio of the differences in the thermal effusivities of the simulated materials was extremely large (urethane:copper, 850:1), participants were not able to discriminate between them. An earlier study with the same set of simulated materials revealed that participants could discriminate reliably between two materials presented to the index fingers when the ratio of their thermal effusivities exceeded 3 (Yang et al., 2008). The decline in performance in the present experiment probably reflects the smaller surface area stimulated (approximately $55 \mathrm{~mm}^{2}$ per stimulus) and the limited spatial acuity for thermal stimuli on the fingertip. The decreases in skin temperature in each region of the fingertip were well above the threshold for discriminating a change in temperature, which, on the thenar eminence of the hand (maintained at $33^{\circ} \mathrm{C}$ ), is $0.11^{\circ} \mathrm{C}$ for cooling stimuli (Stevens \& Choo, 1998).

This result is consistent with other experiments on thermal spatial acuity in which it has been shown that the ability to resolve changes within a region of stimulation is poor (Darian-Smith \& Johnson, 1977). If the skin is warmed or cooled as a result of touching an object, then the location and extent of the thermal stimulus can be determined on the basis of the tactile input. However, in the present experiment, the concomitant tactile input did not facilitate thermal discrimination, and participants were

Table 2

Group Mean Percentage of Correct Responses in Identifying the Cooler Side When Two Simulated Materials Were Presented

\begin{tabular}{lccccc}
\hline & Urethane & Wood & Glass & SS & Copper \\
\hline Urethane & & & & 33.3 & 25.0 \\
Wood & & & 37.5 & 20.8 & 29.2 \\
Glass & & & 16.7 & 41.7 \\
Stainless steel (SS) & & & & & 25.0 \\
Copper & & & & &
\end{tabular}




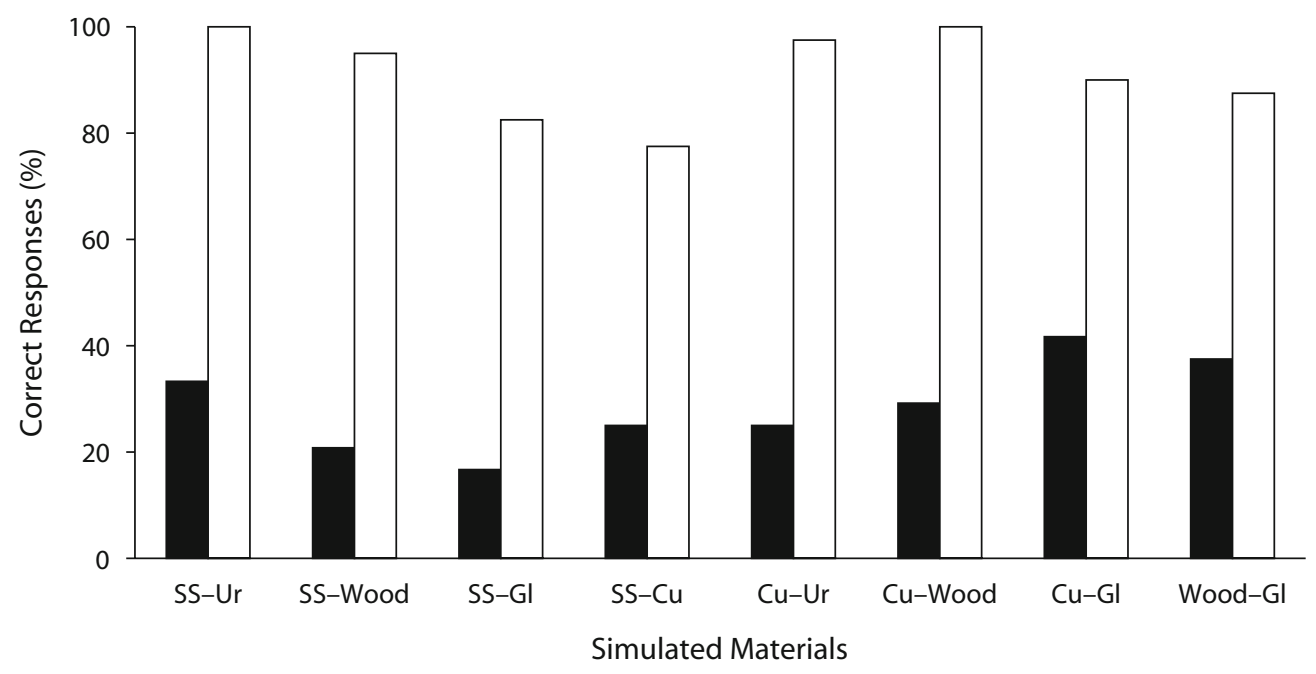

Figure 4. Percentage of correct responses when discriminating the cooler of two simulated materials presented to a single finger (filled bars) or to two fingers (unfilled bars). SS, stainless steel; Ur, urethane; $\mathrm{Cu}$, copper; Gl, glass. Note-The unfilled bars represent data from "Use of Simulated Thermal Cues for Material Discrimination and Identification With a Multi-Fingered Display," by G. Yang, Presence, 17, 29-42. Copyright 2008 by MIT Press. Adapted with permission.

unable to resolve the spatial features of the thermal stimuli on the fingertip. The thermal spatial acuity of the fingertips stands in contrast to their remarkable tactile spatial acuity, which has been estimated to be as low as $0.06 \mu \mathrm{m}$ (LaMotte \& Srinivasan, 1991).

\section{EXPERIMENT 2 Thermal Spatial Summation}

The objective of Experiment 2 was to determine the effect of thermal stimulation of adjacent fingers on the ability to discriminate a change in temperature on a target finger, using thermal stimuli that are typical of those encountered during haptic exploration of objects. One important feature of thermal sensory processing that distinguishes it from other sensory modalities is its focus on registering the quantity of stimulation, regardless of the precise pattern of spatial and temporal stimulation on the skin (Stevens, 1991; Stevens \& Marks, 1979). This means that as the area of thermal stimulation increases, there is a change in the perceived intensity of the stimulus with little perceived gradation in the area of stimulation. For warm stimuli, the area of stimulation and its intensity are equally important in determining thresholds (Kenshalo, Decker, \& Hamilton, 1967), whereas for cold stimuli, the area of stimulation appears to be less important. Although even for cooling stimuli, the threshold is halved with a fourfold increase in stimulation area (Berg, 1978).

In Experiment 2, three fingers on one hand (index, middle, and ring) were stimulated by the same thermal cue while another thermal stimulus was presented to the middle finger on the other hand. The influence of concurrent stimulation of the adjacent fingers on the ability to discriminate changes in the temperatures of the middle fingers of the two hands was studied. It was predicted that as the number of fingers stimulated increased, the ability to discriminate between the thermal cues presented to the middle fingers would change as a function of the fingers stimulated.

\section{Method}

Participants. Eight normal, healthy individuals ( 7 men and 1 woman) 23-30 yearsm of age participated in this experiment. Six of the 8 participants had also participated in the first experiment. The time between the experiments was at least 2 months. The participants had no known abnormalities of their tactile or thermal sensory systems and had no history of peripheral vascular disease. This research was approved by the KAIST Ethics Committee.

Apparatus. The simulated thermal cues calculated from the semi-infinite body model were displayed on the surface of Peltier thermoelectric modules using the same initial conditions (skin temperature $32^{\circ} \mathrm{C}$ and room temperature $24^{\circ} \mathrm{C}$ ) as in the first experiment. The same five simulated materials were also used: urethane, wood, glass, SS, and copper. The cues were displayed on one and three of the Peltier thermoelectric modules, as shown in Figure 3.

Procedure. Each simulated material was paired with itself (five stimulus pairs), and four additional pairs of dissimilar materials were used (urethane-wood, wood-glass, glass-SS, and SS-copper), giving a total of nine stimulus pairs. On each trial, one of these pairs was randomly presented, with one stimulus being presented to only the middle finger on one hand, and the other stimulus being presented simultaneously to the index, middle, and ring fingers on the other hand. For two of the dissimilar pairs of materials (urethanewood and SS-copper), the "cooler" material was simulated on three fingers, and for the other two pairs (wood-glass, glass-SS), the "cooler" material was presented to one finger. The nine pairs of simulated materials were each presented four times, giving a total of 36 trials per hand and 72 trials in total. The order of presentation of each block of 36 trials was randomized.

Participants placed their fingers on the thermal display and, after a sound cue, one of the nine pairs of simulated materials was randomly displayed. A $2 \mathrm{AFC}$ method was used in which participants were instructed to choose which middle finger (left or right) felt cooler. Participants were instructed to focus on only the thermal sensations arising from the middle fingers and to keep their fingers on the display throughout the stimulation period. After $10 \mathrm{sec}$, 


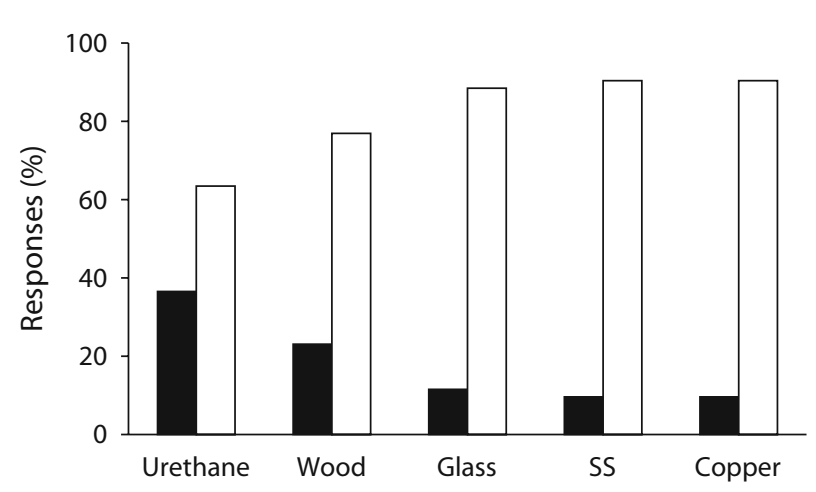

Figure 5. Percentage of responses choosing the cooler side when the same simulated material was presented to one finger (filled bars) and three fingers (unfilled bars) simultaneously. Stainless steel, SS.

they removed their fingers from the display and indicated their responses. There was a 3-min break after every block of nine trials, and participants could request a break whenever necessary during the experiment.

The contact areas of the fingers were measured to quantify the change in surface area from one to three fingers. Participants were instructed to make contact with the surface of the Peltier devices in the same manner that they would use during the experiment. The fingers were moistened, and the surfaces of the Peltier devices were coated with baking soda. After the participants made contact with the Peltier devices, photographs were taken of each fingertip on which the contact area was demarcated by baking soda. Graphical analysis (using MATLAB) was conducted to calculate the surface contact area (Yang et al., 2008).

\section{Results}

The contact areas were calculated from the images taken of each person's fingers. For the index finger, the mean contact area was $100.92 \mathrm{~mm}^{2}$, and for the middle and ring fingers, the mean areas were 100.33 and $100.74 \mathrm{~mm}^{2}$, respectively. There is, therefore, a threefold increase in contact area when thermal cues are presented to three as compared with one finger.

The area of thermal stimulation did affect the perception of thermal changes on the target finger. Concurrent thermal stimulation of fingers adjacent to the stimulated finger influenced the perception of that finger's thermal response. When the same simulated material was presented to the middle fingers of both hands, concurrent stimulation of the index and ring finger, on one hand resulted in the middle finger on that hand being perceived as cooler than that on the other hand, even though the two middle fingers received the same thermal stimulus. The percentages of "cooler" responses for the same simulated material pair as a function of the number of fingers stimulated by the thermal display are illustrated in Figure 5. If thermal stimulation of the adjacent fingers on one hand had no effect on perception, then the percentage of responses for each hand would be at chance - that is, around $50 \%$. A repeated measures ANOVA with the simulated material, number of fingers, and hand as factors indicated that there was a significant effect of the number of fingers $[F(1,7)=112.01, p<.001]$, but no effect of hand or material. On average, participants chose the hand that had three fingers concurrently stimulated on $83 \%$ of the trials, and this increased with the decrease in temperature induced by the simulated material.

When dissimilar simulated materials were presented to the fingers, the effect of concurrent thermal stimulation of the adjacent fingers depended on the cues being presented as shown in Figure 6. When the "cooler" material was displayed on three fingers, performance improved $(89.3 \%$ as compared with $80 \%)$ in comparison with that measured when only one finger was stimulated on each hand (Yang et al., 2008). However, when the cooler material was displayed on a single finger and the "less cool" material was presented to three fingers, the ability to discriminate between the thermal cues displayed on the

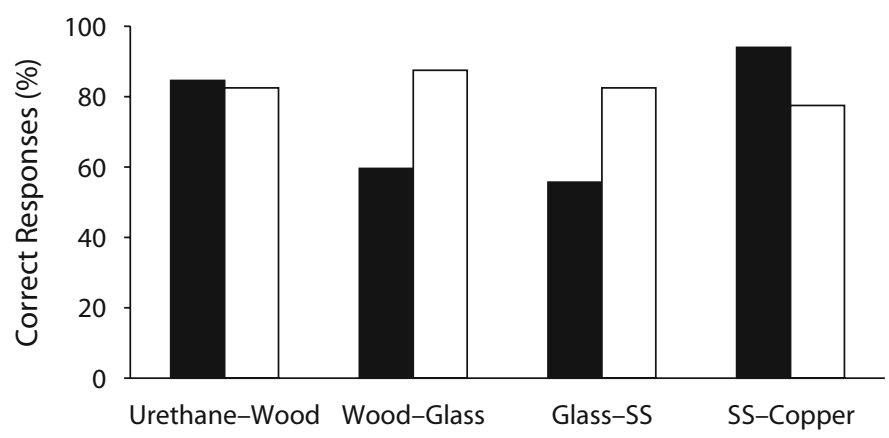

Figure 6. Percentage of correct responses when choosing the "cooler" of two simulated materials in the present experiment (black bars) and when the two materials were presented to one finger on each hand (white bars). In the present experiment, for the urethane-wood and stainless steel (SS)-copper pairs the "cooler" material was presented to three fingers, and for the wood-glass and glass-SS pairs, the "cooler" material was displayed on one finger. Note-The unfilled bars represent data from "Use of Simulated Thermal Cues for Material Discrimination and Identification With a Multi-Fingered Display," by G. Yang, L. A. Jones, and D. Kwon, Presence, 17, 29-42. Copyright 2008 by MIT Press. Adapted with permission. 
two middle fingers deteriorated, in comparison with the single-finger case (57.6\% as compared with $85 \%)$. For these simulated material pairs (wood-glass, glass-SS), participants were unable to discriminate reliably the cooler of the two stimuli presented, and the mean scores were below the threshold level of $72 \%$. These results suggest that the thermal inputs from the three fingers summated, which facilitated performance when the inputs were associated with the cooler simulated material and impaired discrimination when they resulted in a smaller difference between the perceived temperatures of the middle fingers of the two hands.

\section{Discussion}

One of the objectives of this experiment was to examine whether the ability to discriminate which middle finger was cooler changed as the adjacent fingers on one hand were also stimulated. The thermal cues presented were similar to those encountered when the hand makes contact with an object at room temperature. The thermal display that presented the thermal cues was used to stimulate one finger on one hand and three fingers on the other hand simultaneously. The results indicated that when the same thermal cues were presented to the middle fingers of each hand, concurrent thermal stimulation of the index and ring fingers influenced the perception of the thermal changes on that hand. It appears that the thermal inputs summated across the three fingers stimulated, with the result that the middle finger on that hand felt cooler than that on the other hand. When the simulated materials were different, the effect of concurrent stimulation of the adjacent fingers depended on the cues being presented. Discrimination improved when the "cooler" of two simulated materials was presented to three fingers, but it was more difficult if the "less cool" (defined in terms of the predicted changes in skin temperature) of the two materials was presented to three fingers. In the latter condition, participants were unable to discriminate reliably between the simulated materials - a task that could be accomplished quite easily when only one finger of each hand was stimulated (Yang et al., 2008).

These results suggest that spatial thermal summation can occur when noncontiguous areas of the hand are stimulated, and that this can facilitate the discrimination of thermal stimuli. Spatial summation for cold has been demonstrated for both threshold and suprathreshold stimuli (Greenspan \& Kenshalo, 1985; Stevens \& Marks, 1979). These summation effects have also been shown to occur when bilateral and symmetrical sites on the body are cooled. Rózsa and Kenshalo (1977) found that detection thresholds for cooling were significantly lower when both forearms were cooled than when the stimulus was delivered to just one arm. Similarly, magnitude estimation studies have shown that as the area of skin cooled increases, the perceived magnitude of the cold sensation increases as a function of contact area, regardless of the degree of cooling (Stevens \& Marks, 1979). On the basis of these findings, it would appear that in the present experiment, when the neighboring fingers were stimulated simultaneously, the perceived magnitude of the thermal changes on that hand increased, and that this facilitated discrimination when the cooler stimulus was presented to three fingers on one hand. The finding that sensory stimulation of one finger influences the perception of events at other fingers has also been noted in the tactile modality. For example, when the thumb and index finger are simultaneously used to explore a textured surface, the magnitude of the roughness perceived with the index finger is greater when two fingers are stimulated than when the index finger is stimulated on its own (Verrillo, Bolanowski, \& McGlone, 1999).

There is a linear increase in contact area as the number of fingers stimulated increases from one to three. The results from a previous experiment suggest that there may be a ceiling effect for thermal spatial summation across the fingers at a contact area of approximately $300 \mathrm{~mm}^{2}$. Using a material identification task, Yang et al. (2008) found that the ability to identify a simulated material improved if the thermal cues were presented to more than one finger, but that there was no further enhancement in performance if the cues were presented to five-as compared with three - fingers. In that experiment, the contact area increased from an average of $320 \mathrm{~mm}^{2}$ with three fingers to $530 \mathrm{~mm}^{2}$ when five fingers were stimulated. On the thenar eminence of the hand, a ceiling for thermal spatial summation has been suggested to exist at a contact area of around $720 \mathrm{~mm}^{2}$ (Greenspan \& Kenshalo, 1985).

These spatial thermal summation effects are consistent with the results from other studies of spatial processing in the thermal sensory modality. The thermal referral and enhancement effects described by Green $(1977,1978)$, in which thermal stimuli delivered to the index and ring fingers influence the perception of temperature changes at the middle finger, also demonstrate how thermal cues from the fingers summate across the hand. As was observed in the present experiment, when the adjacent fingers are concurrently stimulated with the middle finger, the perceived magnitude of a cold thermal stimulus delivered to the middle finger is greater than when it is stimulated alone (Green, 1977).

\section{Conclusions}

The goal of the present set of experiments was to investigate spatial aspects of thermal processing in the hand in the context in which they are used for haptic object recognition. The results from the first experiment indicate that the thermal spatial acuity of the fingertip is poor, in marked contrast to its remarkable tactile spatial resolution. Even when relatively large thermal differences were stimulated - such as those associated with making contact with urethane and copper-participants were unable to resolve the difference between the two thermal transients. In the second experiment, there was strong evidence of spatial summation across the fingers, which enhanced the ability to discriminate between simulated materials when the cooler stimulus was presented to three fingers. However, when the same stimulus was presented to the two hands, simultaneous stimulation of the two adjacent fingers on one hand led to an enhancement in the perceived thermal response of that hand. Simulated thermal cues can be used to facilitate the discrimination and identification 
of objects, but the spatial thermal processing characteristics of the hand must be taken into account in designing thermal displays that present these cues.

\section{AUTHOR NOTE}

This work was supported by TSI-ITE (Immersive Tangible Environment) (KIST) and the U.S. Army Research Laboratory under Cooperative Agreement DAAD19-01-2-0009. Address correspondence to D.-S. Kwon, Korea Advanced Institute of Science and Technology, HumanRobot Interaction Research Center, 373-1 Guseong Yuseong, Daejeon 305-701, Korea (e-mail: kwonds@kaist.ac.kr).

Note-Accepted by the previous editorial team, when Thomas H. Carr was Editor.

\section{REFERENCES}

Benali-Khoudja, M., Hafez, M., Alexandre, J.-M., \& Kheddar, A. (2003). Thermal feedback interface requirements for virtual reality. In I. Oakley, S. O'Modhrain, \& F. Newell (Eds.), Proceedings of the 2003 EuroHaptics Conference (pp. 438-443). Paris: EuroHaptics Society.

BERG, S. L. (1978). Magnitude estimates of spatial summation for conducted cool stimuli along with thermal fractionation and a case of secondary hyperalgesia. Unpublished doctoral dissertation, Florida State University.

Bergamasco, M., Alessi, A. A., \& Calcara, M. (1997). Thermal feedback in virtual environments. Presence, 6, 617-629.

Businger, J. A., \& BuettNer, K. J. K. (1961). Thermal contact coefficient (A term proposed for use in heat transfer). Journal of Meteorology, 18, 422

Caldwell, D. G., \& Gosney, C. (1993). Enhanced tactile feedback (tele-taction) using a multi-functional sensory system. Proceedings of the IEEE International Conference on Robotics \& Automation, $\mathbf{1}$, 955-960.

Caldwell, D. G., Tsagarakis, N., \& Wardle, A. (1997). Mechano thermo and proprioceptor feedback for integrated haptic feedback. Proceedings of the IEEE International Conference on Robotics \& Automation, 3, 2491-2496.

Darian-SMith, I., \& Johnson, K. O. (1977). Thermal sensibility and thermoreceptors. Journal of Investigative Dermatology, 69, 146-153.

Deml, B., Minalyi, A., \& Hannig, G. (2006). Development and experimental evaluation of a thermal display. In Proceedings of the 2006 EuroHaptics Conference (pp. 257-262). Paris: EuroHaptics Society.

Green, B. G. (1977). Localization of thermal sensation: An illusion and synthetic heat. Perception \& Psychophysics, 22, 331-337.

GreEN, B. G. (1978). Referred thermal sensations: Warmth versus cold. Sensory Processes, 2, 220-230.

Greenspan, J. D., \& Kenshalo, D. R. (1985). The primate as a model for the human temperature-sensing system: II. Area of skin receiving thermal stimulation. Somatosensory Research, 2, 315-324.

Ho, H., \& Jones, L. A. (2006a). Contribution of thermal cues to material discrimination and localization. Perception \& Psychophysics, 68, 118-128.

Ho, H., \& Jones, L. A. (2006b). Thermal model for hand-object interactions. In Proceedings of the Symposium on Haptic Interfaces for Virtual Environment and Teleoperator Systems (pp. 461-467). Los Alamitos, CA: IEEE Computer Society Press.

Ho, H., \& JoNES, L. A. (2007). Development and evaluation of a thermal display for material identification and discrimination. ACM Transactions on Applied Perception, 4, 1-24.

InCropera, F. P., \& DeWitt, D. P. (1996). Fundamentals of heat and mass transfer. New York: Wiley.

Ino, S., Shimizu, S., Odagawa, T., Sato, M., Takahashi, M., Izumi, T., \& IFUKUBE, T. (1993). A tactile display for presenting quality of materials by changing the temperature of skin surface. In Proceedings of the 2nd IEEE International Workshop on Robot \& Human Communication (pp. 220-224). Piscataway, NJ: IEEE Press.

JONES, L. A., \& BERRIS, M. (2003). Material discrimination and thermal perception. In Proceedings of the 11th Symposium on Haptic Interfaces for Virtual Environment and Teleoperator Systems (pp. 171178). Los Alamitos, CA: IEEE Computer Society Press.

JoNes, L. A., \& Ho, H. (2008). Warm or cool, large or small? The challenge of thermal displays. IEEE Transactions on Haptics, 1, 53-70.

Kammermeier, P., Kron, A., Hoogen, J., \& Schmidt, G. (2004). Display of holistic haptic sensations by combined tactile and kinesthetic feedback. Presence, 13, 1-15.

Kenshalo, D. R., Decker, T., \& Hamilton, A. (1967). Spatial summation on the forehead, forearm, and back produced by radiant and conducted heat. Journal of Comparative \& Physiological Psychology, 63, 510-515.

LAMotTe, R. H., \& SRINIVASAN, M. A. (1991). Surface microgeometry: Tactile perception and neural encoding. In O. Franzen \& J. Westman (Eds.), Information processing in the somatosensory system (pp. 4958). London: Macmillan.

Matweb (2007). Database of material properties. Retrieved June 2007, from www.matweb.com.

Rózsa, A. J., \& Kenshalo, D. R. (1977). Bilateral spatial summation of cooling of symmetrical sites. Perception \& Psychophysics, 21, 455-462.

Stevens, J. C. (1991). Thermal sensibility. In M. A. Heller \& W. Schiff (Eds.), The psychology of touch (pp. 61-90). Hillsdale, NJ: Erlbaum.

Stevens, J. C., \& ChOo, K. K. (1998). Temperature sensitivity of the body surface over the life span. Somatosensory \& Motor Research, $15,13-28$

Stevens, J. C., \& Marks, L. E. (1979). Spatial summation of cold. Physiology \& Behavior, 22, 541-547.

Verrillo, R. T., Bolanowski, S. J., \& McGlone, F. P. (1999). Subjective magnitude estimate of tactile roughness. Somatosensory \& Motor Research, 16, 352-360.

Yamamoto, A., Cros, B., Hashimoto, H., \& Higuchi, T. (2004). Control of thermal tactile display based on prediction of contact temperature. Proceedings of the IEEE International Conference on Robotics \& Automation, 2, 1536-1541.

YANG, G., Jones, L. A., \& Kwon, D. (2008). Use of simulated thermal cues for material discrimination and identification with a multifingered display. Presence, 17, 29-42.

YAnG, G., KyUnG, K., JeOng, Y., \& Kwon, D. (2005). Novel haptic mouse system for holistic haptic display and potential of vibrotactile stimulation. In Proceedings of the 2005 IEEE/RSJ International Conference on Intelligent Robots and Systems (pp. 1109-1114). Piscataway, NJ: IEEE Press.

Yovanovich, M. M. (1986). Recent developments in thermal contact, gap and joint conductance theories and experiments. Proceedings of the 8th International Heat Transfer Conference, 1, 35-45.

(Manuscript received September 12, 2007; revision accepted for publication July 17, 2008.) 